\title{
An Empirical Study of Cognition and Theatrical Improvisation
}

\author{
Brian Magerko, Waleed Manzoul, Mark Riedl, Allan Baumer, Daniel Fuller, Kurt Luther, and \\ Celia Pearce
}

Adaptive Digital Media Lab

Georgia Institute of Technology

686 Cherry St.

Atlanta, GA 30332-0165

\{magerko, w.manzoul, riedl, abaumer3, dfuller3, luther, celia.pearce \}@gatech.edu

\begin{abstract}
This paper presents preliminary findings from our empirical study of the cognition employed by performers in improvisational theatre. Our study has been conducted in a laboratory setting with local improvisers. Participants performed predesigned improv "games", which were videotaped and shown to each individual participant for a retrospective protocol collection. The participants were then shown the video again as a group to elicit data on group dynamics, misunderstandings, etc. This paper presents our initial findings that we have built based on our initial analysis of the data and highlights details of interest.
\end{abstract}

\section{Author Keywords \\ Improvisation, empirical study, cognition, drama, narrative, performance}

\section{ACM Classification Keywords}

J.5: Computer Applications: Arts and Humanities.

\section{General Terms}

Experimentation.

\section{INTRODUCTION}

Research on the formal study of cognition and creativity has covered diverse creative domains like music performance $[22,24,25]$, painting and dance as well as traditionally non-artistic domains like scientific discovery [3] and business management [16]. This work has included investigations into the cognitive processes that are applied during human creative acts $[12,24,25]$. This research includes not only attempts to reach a better understanding of an important facet of the human experience, but also has attempted to codify these processes for creating computational creative works (e.g.

Permission to make digital or hard copies of all or part of this work for personal or classroom use is granted without fee provided that copies are not made or distributed for profit or commercial advantage and that copies bear this notice and the full citation on the first page. To copy otherwise, or republish, to post on servers or to redistribute to lists, requires prior specific permission and/or a fee.

C\&C'09, October 26-30, 2009, Berkeley, California, USA.

Copyright 2009 ACM 978-1-60558-403-4/09/10...\$10.00.
$[8,29])$.

Improvisation is a relatively understudied aspect of creativity and cognition. One way of viewing improvisation is as the act of real-time dynamic problem solving [12]. One of the most recognizable manifestations of improvisational problem solving comes from the theatre arts community. Improvisational theatre - or simply improv - is a rich source of data for reaching a better understanding of improvisational problem solving and cognition $[11,32]$. This is in part due to the diversity of performative activities in improv, which allows us to manipulate independent variables for purposes of experimentation, and the decoupling from real-world problems (e.g., emergency management) that are hard to control or recreate. Focusing on improv theatre, we can more specifically define improvisation as a creative act to be the "creation of an artifact and/or performance with aesthetic goals in real-time that is not completely prescribed in terms of functional and/or content constraints." Our definition here intentionally focuses on the process of creating; improvisation is viewed as an active endeavor that is equally, or more, important than the final product. That is, how you get to an outcome is more important than the outcome.

Improv theatre is relatively unexplored from a cognitive scientific perspective. Modern improv theatre, born out the Commedia dell'arte tradition, typically involves a troupe of actors that perform short scenes based on "games" that are selected for them. Games typically involved telling the actors rules for what they can / cannot do in a scene and a suggestion from the audience. Improv theatre is a remarkable example of creative group performance that differentiates itself from other improvisational forms (e.g. jazz) because (a) the creation process of narrative content takes place entirely in realtime, (b) there is no explicit coordination between the actors, and (c) in the case of unscripted improv, the constraints on a performance are typically of the form of a set of game rules plus audience suggestions, both of which are typically unknown to the performers until right before a scene is performed. 
The goal of the research presented here is to acquire a deeper understanding of human creativity and cognition through an empirical study of improv. By conducting a large-scale study of performing human improvisers, we hope to build an understanding of the cognitive processes of both novices and experts when improvising a scene on stage. Once we have built an empirically based theory, we can then apply that theory to the creation of digital improvisational actors that can be used in a variety of contexts (e.g. computer games, educational media, intelligent interfaces, etc.).

Our interest in building computational models of improvisation has led us to focus on specific aspects of cognition, which are reported in this paper, and eschew investigating others (e.g. natural language interaction, body language, and cultural knowledge use). The domains we have set aside from now are difficult to represent computationally compared to the more core areas reported here (e.g. shared mental models, reasoning about goals, and improvisation strategy use). Future work will attend to these areas, but were not deemed as immediately useful for creating digital actors.

This paper reports our methodology used for studying improvisers and the current overall theory that has been generated from our data collection efforts with examples from the data.

\section{RELATED WORK}

\section{Creativity Research}

Creativity has been a topic of interest to philosophers, psychologists, and computer scientists. Each has its own notion of what creativity is. However, virtually all definitions of creativity emphasize the notion that something new comes into existence and that this new thing is somehow valuable to the individual and to society in general. For example, Boden [3] defines creativity as "the ability to create ideas or artifacts that are new, surprising, and valuable" (pp. 1). Johnson-Laird [12] hypothesizes that there are three types of algorithms that can produce creative results: (a) Neo-Darwinian, in which recursive algorithms generate new results through arbitrary processes on existing elements and then evaluate; (b) Neo-Lamarckian, in which discovery of new elements is guided by the application of the evaluation criteria; and (c) a hybrid approach, in which generation occurs with some criterion guidance but is also capable of producing results that do not fit all constraints and must be evaluated separately. Johnson-Laird suggests that the hybrid approach is most similar to the way the mind works and applies this analysis to jazz improvisation.

Creativity is most often studied in the context of a particular type of creative artifact or activity. Any computer system that appears to create a novel artifact or concept can be considered creative [30]. However, most research in computer systems that appear creative focuses on the parameters of the artifact or concept that make it creative. For example, story generation algorithms may use planning algorithms because multi-agent plan structures resemble plot structures [25]. Likewise, previous work on improvisational theatre [9] uses reactive planning to assemble and combine pre-scripted activities. However, research into creative computer systems that will generalize requires investigation of creativity from the perspective of the cognitive processes that are utilized by human practitioners.

\section{Improvisation Research}

There are added constraints to improvisational creative works that make them a unique problem within the space of creativity. The current body of research on improvisation, which most notably comes from the improvisational music domain, points to the following generalities:

Improvisation is a constant process of receiving new inputs and producing new outputs [22]. Improvisational dance, theatre, music, etc. all depend on performers observing their own and other performers' actions, executing some quick deliberative process, and then selecting new actions to perform. An improvisation model must be able to process and interpret these inputs as knowledge to be involved in decision-making.

Improvisation is a "continuous and serial process" [15, 16] as opposed to one that is "discontinuous and involving iteration," such as music composition [28]. This suggests that there are specific cognitive processes that are employed during improvisation that are either a) different from those used during non-improvisational acts or b) are used with different constraints than those used during non-improvisational acts.

Improvisation involves decision-making based on domain-specific as well as real-world knowledge [24, 35, 36]. A key aspect of this knowledge is the use of a "referent" as background knowledge for improvisation $[16,22]$. A referent eases cognitive load during improvisation by providing material for variation, allows for a palette of pre-performance structures to be created by the performer, and reduces the need for complex communication cues with other performers. Therefore, a model of improvisation should be capable of processing and applying these kinds of semantic structures for both inspiration and the lessening of cognitive load.

Collaborative improvised pieces (as opposed to solo works) may involve both explicit and implicit communication $[1,24]$. Body language, domain-specific cues, and verbal commands all contribute to the collaborative process of performing a group act. Any model of improvisation needs to address how communication to others in the group is used for coordination.

Improvisation is a process of severely constrained human information processing and action [12, 22, 37]. As Pressing [22] points out, musical improvisers must, in real-time, optimally allocate attention, interpret events, 
make decisions about current and future actions, predict the actions of others, store and recall memory elements, correct errors, control physical movements, and integrate these processes seamlessly into a performance. It remains to be seen if this view of cognitive constraints maps on to the theatre improv domain however, this research takes a significant step toward addressing this question.

\section{IMPROVISATION IN ARTISTIC DOMAINS}

Based on some of the research cited above, we have developed a working definition of improvisation in artistic domains, such as improvisational theatre, to be the "creation of an artifact and/or performance with aesthetic goals in real-time that is not completely prescribed in terms of functional and/or content constraints." This definition intentionally focuses on the process of creating; improvisation is viewed as an active endeavor that is related to, but not defined by, the resulting creation. The inclusion of aesthetic goals is intended to limit the discussion to artistic fields as opposed to the kind of improvisation that is practiced in traditionally non-artistic fields (e.g. engineering or sports).

The idea of the performance occurring in "real-time" is a difficult aspect to pin down formally. While a jazz musician is improvising, they may rely on previously stored memories to create their current solo or even pause for a moment to reflect on their solo before deciding what to do next [24]. Even though they are not "spontaneously" creating an entire solo without relying on things like previous memories or looking ahead, it would be hard to argue that they are not improvising the music. This inclusion of "real-time," therefore, is intended to indicate that a process of revising the final product is not possible. When coupled with the notion of improvisation as a performative act, we thus define it as a creative effort that excludes traditional composition or practicing a performance. This definition could reasonably be made more precise by including the requirement of an audience. The last feature of our definition, that the act is not "completely prescribed in terms of functional and/or content constraints," infers that a non-improvisational performance within any art form is decided beforehand in terms of the form (e.g. the style, key signatures, time signatures, etc. used in a musical piece) and content (e.g. the notes for a musical piece, the lines for a play, etc.). An improvised performance is therefore one that is not fully specified along one or both of these dimensions. For example, a jazz tune may have a specific key and chord changes written for the solo section, but the individual notes that are played within those predetermined constraints are wholly left up to the soloist. This definition is not intended to be a unified definition across all interpretations of improvisation, but is intended to limit the scope of possibilities to the specific phenomena that we are trying to observe.

We wish to acquire a deeper understanding of human creativity as a cognitive process. Improv becomes a tool for more rigorously exploring the phenomenon of improvisational problem solving on a larger scale. In the following sections, we describe our methodology for collecting and interpreting data from improv theatre performers.

\section{METHODOLOGY}

Research Participants

The research participants were recruited from a pool of local improv troupes ranging in experience from novice to expert. The inclusion of a broad spectrum of troupe experience levels will allow us to do future comparisons between experts, intermediate, and novice improvisers. Atlanta, the city where the study was conducted, has a strong improv theatre community. This helped in both identifying and recruiting groups to participate in our experimental sessions. All of the participations were over 18 , had at least some experience in performing theatrical improvisation, and were reimbursed monetarily and with dinner provided for the evening sessions.

\section{Empirical Study Design}

Previous work on studying improvisation has mainly focused on observational or informal interview techniques $[15,24]$. Our approach to studying theatrical improvisation has been designed to collect the most detailed data possible on the cognitive processes employed. We would ideally be able to directly observe the processes employed during performance, but direct instrumentation to collect cognitive data would be both invasive to the performers and interfere with the performance itself. Because improvisational theatre is highly social, we also believe that it is vital to analyze cognition not only at the individual level but also at the group level. We hypothesize that group dynamics are highly important to improvisational acting, so a proper experiment must attempt to capture data related to group decision making, social relationships, familiarity, etc. Therefore, we have designed an experimental methodology for studying cognition in improvisation that focuses on observational data, individual cognitive data, and group dynamics.

Our experiment design puts our participants through four separate stages, each targeted at one or more specific data types (described in the Initial Findings section below): pre-performance survey, performance (which includes a warm-up period), individual retrospective protocol collection, and group retrospective protocol collection. The three stages are typically repeated 2-3 times during an experimental session, allowing us to collect varied data and to control different variables from the same group of performers.

Stage 1: Pre-Performance

In the pre-performance stage of the experiment, participants are asked to fill out a written questionnaire that asks questions about their level of expertise and experience with the troupe. Expertise is determined not by self-identification, but rather by specific metrics, such as 
training, period for which the person had been doing improv, and whether or not they have done so professionally (defined as receiving regular payment for performances). Subjects are also asked questions about their history and relationship with the troupe with which they were performing.

Stage 2: Performance

During the performance stage, participants are led to a performance area, allowed to do whatever warm ups they typically use to get prepared, and are then given an improv "game" to perform. Improv games are typically comprised of a functional constraint, which directs the actors to perform a scene in a certain way (e.g. "You can only speak in questions for the entire scene.") and a content constraint (e.g. "You are both plumbers at a zoo."). Actors are typically given a time constraint for how long the scene can last. When the time constraint is reached, an experimenter will call the end of the scene.

We have modified several established improv games (i.e. traditional functional constraints for scenes) and predetermine content constraints for the purpose of eliciting specific kinds of data. For example, one game, which is simply named Game, has the point of simply "creating a scene" (not necessarily a comedic one) in accord with some content suggestions. This has been modified into three separate versions to allow us to study narrative development:

- No content constraints -- the actors are instructed to perform a scene with no additional constraints provided;

- Low content constraints -- the actors are instructed to perform a scene and are given typical content constraints (i.e. a location and a relationship between the characters); and

- High content constraints -- the actors are instructed to act out a very specific plot structure with specific characters involved. This game is intended to elicit data on how actors construct a story, employ content constraints to guide the decision making process, and communicate goals, among other things.

Our other games are called Party Quirks and Film and Theatre Styles. Party Quirks involves four players, the party host and three guests. Each guest has a special "quirk" suggested by the audience. Everyone in the performance space, including the audience, knows the guests' quirks except the host, whose job it is to guess them throughout the course of the scene. This game was chosen to study group dynamics and how improvisers communicate knowledge. Film and Theatre Styles is typically for two actors, who are given a scene (e.g., "two men painting a bridge") to perform in different film and theatre styles (e.g. "film noir") that are changed throughout the course of the performance. This game was chosen to help us study the use of semantic knowledge and narrative development. It is also the only two person game we play. Each performance is videotaped and uploaded to a video-streaming server where the video can readily be used for stages 3 and 4 of the experiment.

Stage 3: Individual Retrospective Protocol

Each participant is taken to a private room for an individual retrospective interview, each of which is videotaped. The purpose of this interview is to learn as much as possible about what the participants were thinking about during the performance. Video capture from the performance is played back to the participant, with the interviewer stopping the video at short intervals to allow the participant to describe what they were thinking about during each action in the scene. Per a typical retrospective protocol collection, improvisers are asked to describe what they were thinking at the time of the activity. They are specifically asked not to critique their performance, nor to concern themselves with their thoughts after the performance concluded. Rather, they are prompted at each phase of the performance to discuss their thoughts in the moment each action was being performed.

\section{Stage 4: Group Interview}

The performers are brought back together in a separate room for a group interview. The purpose of this interview is to explore aspects of group dynamics and decisionmaking. Since improvisational theater relies heavily on performers interpreting and understanding each others' performances, the group interview allows participants to unpack what the others were thinking during the performance, and provide insight into how they implicitly come to agree on what was going on in the scene. This interview is often where shared references emerge, such as knowledge of the other actors, past experiences performing with the group, techniques the particular group has learned in its training, or discussions about how participants collectively modeled the scene.

\section{Coding the Data}

The combined data -- the questionnaire, the performance video, the individual interview video, and the group interview video -- are combined for coding using the Anvil software package. We have employed a data driven approach to induce a working theory of cognition in theatrical improvisation. The high-level categories that we have developed are described in the Initial Findings section, along with subcategories that are of particular interest.

\section{Limitations of Methodology}

While this retrospective analysis method has several limitations, of which we were aware, an extensive and rigorous process of both study design and prototyping determined that this was the most appropriate means for gathering the data sought by this research. As mentioned earlier, the use of cognitive measuring instrumentation would be too invasive, and would interfere with the performances; furthermore, data collected in this way could track parts of the brain being used, but not the specific processes they measure, thus rendering the 
findings difficult to implement in a computational model, which is our end goal.

The primary shortcoming of this method is that it relies on the participant's memory and willingness to communicate his or her thoughts. The method of describing activities in-progress has been used previously to successfully analyze various forms of professional practice, such as surgery and architecture [31]. Because real-time reflection of this sort would interrupt the performance process, it was not feasible for this research. However, since improv performers are both verbal by nature and trained to be highly reflective, the retrospective protocol method was extremely effective for the target group. While it might be less effective with improv practitioners in other, lessverbal fields such as dance or music, this method took advantage of inherent reflective verbal communication skills specific to the discipline being studied. Troupes have actually reported incorporating this process into their rehearsal practice, which was a surprising effect of conducting our study.

\section{INITIAL FINDINGS}

The results we have found based on our analysis of observational, retrospective protocol, and group interview data has yielded the following high-level topics:

- Basic Cognition

- Shared Mental Models

- Narrative Development

- Referent Use

There are other possible data groups, such as linguistic production, gesture, posture, or cognitive workload, but our current efforts focus on what we see as a manageable and relevant data set for creating synthetic characters. These other aspects of performance are large endeavors on their own in terms of cognitive science research and would have been incredibly challenging to tackle within the scope of this work.

The decision cycle from Newell's Unified Theory of Cognition [21] has heavily informed our data analysis. When reasonable, cognitive acts are separated into either: receiving input, the elaboration of new knowledge based on other knowledge or inputs, the proposal of new operators / actions / goals to pursue, the selection of one of the proposed courses of action, and the execution of what was decided. We have found this model incredibly useful for constructing our working theory based on this highly rich and large data set. This decision cycle is particularly useful since it is already employed as a key architectural feature in the Soar architecture [21], which we can subsequently use for the construction of our digital improvisers.

The following subsections represent an outline of our current domain theory on the underlying cognition involved in improvisation, including examples from our data set.

\section{Basic Cognition}

We found that improvisers engage in cognitive processes such as inference, schema generation, mental imagery, theory of mind, and decision-making while performing a scene. The following highlights some examples of these processes.

\section{Inference}

Improvisers would frequently report inferring information about the scene from other improviser's actions, specifically about the scene's location and the improviser's goals and knowledge in the scene. For example, this inference occurred early in a game of Party Quirks. Improviser D1, watching the party host, D2, set up the scene, described the following:

“... and then I thought, 'Who is he trying to be?' Like is he having a house party or is he a college guy? Are we in the middle of the forest?' I mean I'm trying to picture where he is setting his party and who his character is. I thought eventually because there was a door [the host pantomimed opening a door], well, we have to be inside then. We have to be in someone's house."

D2 inferred where the party was being held using what he saw from D2's actions. This is an example of theory of mind, which states that one uses a model of others in part to determine how to act in a social environment. D1 later infers information about D2's knowledge in the scene, that D2 knows D3's quirk (D3 was playing someone who was invisible):

"When [D2] said, 'I'll put your stuff right over here' and he walked away and didn't make eye contact with her like as if he didn't see her...I'm like, '[D2] knows [D3]'s invisible.",

D1 observed D2's actions and inferred information about what D2 knew, specifically that D2 knew that D3 is playing someone who is invisible, and kept this information in mind as he prepared to enter the scene.

\section{Schema Generation}

Improvisers reported generating a schema of features of a character's activities and behaviors to be acted on over the course of a scene [2]. Improviser A4 was given the quirk of being addicted to video games in a run of Party Quirks. He reported when he received his quirk,

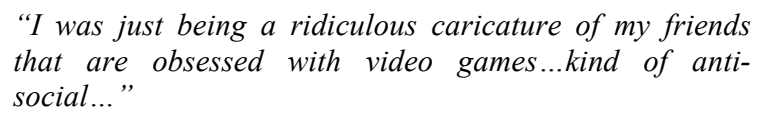

His schema of video game addicts was later reflected in his performance of the quirk; he was rude to the other party guests who interrupted him and demonstrated an intense demeanor. This example is highly related to improvisation games that are used to teach how to build characters, such as Johnstone's Fast Food Stanislavski technique [12].

Mental Imagery

Improvisers reported the use of mental imagery before and during an improvised scene. Improviser A2, during a 
round of Party Quirks reports how mental imagery plays a role in determining future actions:

"Well, I'm trying to listen to everything that they say because if they create something like that punch bowl is right there in the front [the host pantomimed placing a punch bowl onto a table], so you don't want to walk through it later. So you have to pay attention to that."

In general, improvisers visualize their environment and considered how it would affect their actions.

\section{Decision-Making Strategies}

Improvisers have reported using a number of strategies to make decisions about how to move a scene forward. These included using their notions of 'believability' or dramatic interest, using established improvisation techniques such as "Yes, and..." (as discussed in the Referent Use section below), and mimicking another improviser. Two particularly interesting decision-making strategies are detailed below.

Game Within a Scene. Improvisers reported that they would sometimes try to find the "game within the scene," which means to introduce additional constraints, ad hoc, into a scene, to help guide which action to execute next in a scene. Improvisers D5, D6, and D7 played three college friends who have reunited at the zoo in a low-contentconstraint run of Game. D5 and D6 chose to play reckless characters (they both wanted to throw their food into the lions' cage) and D7 chose to play a prudish character that consistently opposed their ideas. D6 attributed his reckless actions to being unmarried and childless and says that he doesn't like D7's wife. D7 reported his thoughts after hearing improviser D6 tell him that he wished he was not married to her:

"So I started thinking like, 'What do I make my wife?' He wishes I didn't have my wife and I'm thinking, 'Should I not like my wife or should I be in love with my wife?' And that's going to base how I feel about what he's saying to me. My opinion about the woman I'm married to. So I could've sided with him and said, which I almost said; I almost said, 'I wish I wasn't married to her either. She's a real [expletive].' But I decided to take the other stance and going like, 'Why? What's wrong with my wife?' and then thinking like, 'I'm completely finding sort of a game within the scene. I'm going to be the total outsider from this trio of friends. I'm going to be the guy whose opinion differs from them, no matter what.",

D7 evaluated previous dialogue of the scene, saw an emergent characteristic of his character, and choose an option of action that was most consistent with this characteristic. This technique appears to be used more often by expert improvisers; future work will focus on this phenomenon in a novice / expert analysis of our data.

Reality of the Scene. An improviser reported considering the "reality of the scene" to choose their next action, which involves reflecting on the current state of the scene. Improvisers D1, D2, D3, and D4 performed a run of Party Quirks in which they played a lost travel agent, the host, someone who was invisible, and a person obsessed with health issues, respectively. D1 was considering his options for his next action in the scene while watching D2 and D4 interact:

"Well, do I go back and watch the fight [D2 had
mentioned that he will leave a boxing match playing on
the television]? Do I go to the keg and get a beer [D2 had
pantomimed tapping a keg of beer] or do I go get some
food that [D3] is eating?' But I remember thinking,
'D3]'s [who is supposed to be invisible] eating and no
one is noticing the fact that these invisible chips [We
believe he meant "floating chips"] are moving.' So that
needs to be addressed, that's the reality of the scene...the
fact that she's invisible and there's food moving."

Later in the scene, D1 moved to the food table and exclaimed that the chips are moving on their own. In this way, D1 proposed a number of options and chose one based on the most pressing issue regarding the reality of the scene.

\section{Shared Mental Models}

Shared mental models are the common framework of knowledge (i.e. mental models) shared among the members of a group [4, 17]. In improvisation, shared mental models usually require effort to create. Sometimes the mental models of a group disagree, and cognitive divergence occurs (i.e. when improvisers have different internal models about what is going on in a scene). These disagreements are resolved through cognitive convergence, which is the process of building towards a goal state of cognitive consensus (i.e. the agreement of assumptions) [19, 20]. When cognitive consensus is reached, mental models are shared among the group (at least partially). Understanding cognitive divergence and cognitive convergence provides insight into the process of how improvisers establish these shared mental models.

\section{Cognitive Divergence}

When the assumptions of two or more improvisers do not match, there is cognitive divergence. For example, improvisers $\mathrm{C} 2$ and $\mathrm{C} 3$ experienced cognitive divergence in one performance when $\mathrm{C} 2$ assumed that he was enacting a female character while $\mathrm{C} 3$ assumed that $\mathrm{C} 2$ was portraying an "effeminate man." In this context, assumption refers to any belief that influences an individual's understanding of the scene, such as assumptions about the audience or the environment [18, 20]. Assumptions can be either diegetic (of the story world of a scene) or non-diegetic (beyond the story world of a scene). A diegetic assumption might concern the personality of another improviser's character ("Character A seems really loud and angry!") while a non-diegetic assumption might concern the rules of a specific improv game ("I thought the audience was supposed to clap when I made a correct guess!").

Divergence of assumptions reflects the disparities between individual mental models. For example, in one scene, improviser D1 began by raking leaves, reportedly imagining that he was somewhere outside. D2 and D3, however, reported that they thought D1 was "sweeping 
up" at a coffee shop. In this case, there is a divergence of environmental assumptions because of the competing mental models of the improvisers involved.

\section{Cognitive Convergence}

Cognitive convergence is the process of establishing cognitive consensus, which is a state of agreement of assumptions between two or more people and is necessary for shared mental models to exist. For example, in one group retrospective interview, improviser D3 mentioned to D1 that "...you reminded me that [Pablo Escobar] was dead by doing that," showing that D1 and D3 had achieved cognitive consensus on the issue of Pablo Escobar's death. Although cognitive consensus is desirable, it is not always achieved. Sometimes improvisers give up before consensus is reached, or they refuse to accept the mental model of others out of stubbornness or intended comedic effect. We describe all of these acts as rejection.

Three steps of cognitive convergence usually occur before cognitive consensus is reached. First is observation, the point at which an improviser realizes that his mental model diverges from others'. Next is repair, which refers to all attempts to reconcile divergences $[6,34]$. Repairs can either be attempted in order for an improviser to align himself with another improviser's mental model or in order for an improviser to align another improviser with his own mental model. The final step of cognitive convergence is acceptance, during which cognitive consensus may occur [6, 34]. It is also possible that consensus may be rejected or that an improviser will achieve perceived cognitive consensus, wherein they think that they have achieved consensus, but actually have not. Finally, when two improvisers reach consensus, there is usually an explicit external acknowledgment that they both understand each other [6, 34].

A good example of this process is in one scene of Party Quirks in which the host, improviser A1, struggled for a long time to guess the character quirk of improviser A2. By this point, A1 had already attempted several repairs by making guesses about A2's quirk. Only after A2 gave A1 a couple of very obvious hints did A1 finally understood that A2 was a kleptomaniac. At this point, consensus existed between A1 and A2 because A1 knew A2's quirk, but divergence also existed between $\mathrm{A} 1$ and $\mathrm{A} 2$ because A2 did not know that A1 knew her quirk. Even though A1 knew A2's quirk, he "was resigned" because "the last thing [he] wanted to have to do was admit that the word was 'kleptomaniac." By rejecting consensus, he allowed divergence to continue. However, A1 eventually told A2 what he thought her quirk was, achieving consensus, and the audience acknowledged that he was correct with applause.

\section{Narrative Development}

We have applied concepts in narrative theory to understand how to categorize what improvisers do related to developing a narrative while on stage. Narrative is can be defined as a story (the content in the narrative) plus its discourse (how that story is related to an audience) [5]. A story's constituents are existents (e.g. people and places) and events (what occurs in a narrative) [5]. Discourse is derived from the narrative's manifestation (the medium chosen) and structure (the organization in which the narration is related) [5]. These concepts will be examined in detail within the following sections.

\section{Story}

Story can be broken into existents and events. Existents are characters, settings, and everything that exists within a scene. Improvisers often reported making decisions related to the characters and environment involved in a scene. Improvisers define character by how they develop traits (e.g. relationships, goals, history, physical and mental attributes) and how they demonstrate verisimilar consistency with those traits. Similarly, environment is the imagined location onstage as well as its objects (e.g. a hammer in a warehouse) and attributes (e.g. cold in a graveyard). The consistency of the environment is also an important aspect of performance. Improvisers often strive to keep track of what objects are created and where.

As opposed to what exists in a scene (existents), events are what occur on stage. Ryan proposes terminology for describing events that matches our data: active and passive events [27]. Active events significantly alter the state (i.e. the current situation on stage [23]) of the scene, while passive events do not. The intention in performing an event does not always affect the state of the scene as desired. In other words, an event that was intended to affect the scene could actually have no effect and vice versa. Therefore, the improviser's reported intentions to affect the scene as well as the actual effect of their actions on the scene are both recorded.

\section{Discourse}

Discourse refers to the manifestation and structure of a narrative [5]. Manifestation is simply the medium used to create the narrative (e.g. ballet, cinema, graphic novel), which is not of particular note since we our only studying one particular manifestation of improvisation - stage performance. The structure of the narrative, on the other hand, is a connected set of narrative statements that relate the story, the order of situations and events, the speed of narration, etc. [23]. In dramatic theatre, the discourse is derived from the dramatic text. This indicates who should speak in turn, who should move where, and the structure of the performance. Improvisational theatre lacks a dramatic text, so its equivalent is created and developed ad hoc by the improvisers on stage. This involves a rapid intake and processing of data [22].

During an improvisational performance, the narrative is simultaneously created and executed by its discourse through a series of offers $[10,33]$. An offer made by an improviser introduces an idea or possible progression to the scene (e.g. When one improviser turns to another and says, "Watch out for that shark," the first improviser is 
offering the idea of a shark being in the scene and the possibility of danger). In order to advance the state of the scene, an improvised scene is constructed through making, accepting, and rejecting offers. Our data consistently suggests that for a scene to progress, the events that alter a narrative's state are active intent (i.e. intending an event to significantly alter the state of the scene) offers that are accepted. However, in some cases, passive intentions (i.e. not intending an event to significantly alter the state of the scene) are misinterpreted as active intentions and that divergence needs to be reconciled (see Shared Mental Models).

\section{Referent Use}

A fundamental tenet of improvisational acting is that improvisers perform in the moment, rather than solely from plans or pre-scripted actions. However, audiences can be easily misled by improvisers' natural and spontaneous behavior into forgetting the importance of years of training and experience that they bring with them and rely upon during a performance. A key artifact of this training and experience is improvisers' use of referents, meaning specific terms or language referring to improv techniques employed or functional / content constraints used within a particular scene or environment. As with many of the performing arts, improvisation claims an extensive vocabulary, a set of widely held core principles (e.g., accept an offer and build on it), and a long list of techniques and games used within scenes.

Our data suggest that this knowledge may affect improvisers' cognitive processes in at least three different ways. First, referents often signal connections between what the improvisers is thinking at a particular moment and past experiences; what has happened to the improviser in similar past performance situations may affect his or her current decision. Second, referents in the current scene, such as game rules or conventions for how you play a particular game, may constrain improvisers, narrowing the space of possibilities to make decisionmaking easier. In his study of jazz improvisers, Pressing refers to these constraints as "processing reduction" [22]. Third, referents provide the means for a group of improvisers to quickly reach a shared mental model of the scene (i.e. a shared understanding of the location, characters, roles etc.) in the highly dynamic, real-time environment of stage performance (Shared Mental Models are discussed in their own section above). When referent use is externalized (i.e. verbally identified and defined by improvisers) during our retrospective protocol analysis sessions, it provides powerful and measurable evidence of its effect on improvisers' performances and thought processes.

The following subsections describe two of our main findings in referent use for improvisational theatre.

\section{Game conventions}

Game conventions refer to expectations that improvisers have developed from their existing knowledge of how to play particular improv games. In contrast to game rules, which are given to improvisers at the start of each game during a performance, game conventions vary from improviser to improviser and seem to be influenced by training (i.e. how they have been taught to play) and experience (how often they have played and which versions of the games). For example, in a run of Party Quirks, the host, B4, guessed B3's quirk early on, which lead B3 to exit the stage for the rest of the run. B3 told us,

"I thought that we weren't supposed to... so in short form, traditionally, games like this, and on Whose Line is it Anyway?, once you're in, you're in, and once you're out, you're out."

Improvisers frequently mentioned game conventions in scenes when their expectations conflicted with what actually happened during a performance. For example, in the same run mentioned above, the host struggled for several minutes to guess B2's quirk, "video game addict", because B2 gave no explicit feedback about the host's guesses of "addict" and "game addict". B2 explained,

"A lot of times, if you do play these kind of games, you have an emcee that would say 'scene' or say 'close enough.",

A divergence from this expectation led to a breakdown in the performance. Thus, game conventions are means by which improvisers make decisions, but they may not transfer well to unfamiliar performance environments or with new scene partners. As others have mentioned [16, $22,29]$, this kind of background knowledge provides material for variation, allows for a palette of preperformance structures to be created by performers, and reduces the need for complex communication cues between performers. When one improviser begins to use a game convention that is known by the others, they immediately know what he or she is trying to accomplish and what kinds of responses are appropriate.

\section{Acceptance}

Halpern et al. [7] write that "[w]hen improvisers meet on stage, they agree to accept each other's initiations; they must completely commit to the reality they create for each other without a moment's hesitation" (p.47). This attitude of complete agreement is also called the "yes, and" rule. Agreement, and "yes, and"-ing, requires that improvisers build off each other's ideas and accept the "offers" made.

We observed improvisers making acceptance a core construct of their performances. They also consistently used the same terminology — "offer" and "yes-and"- to talk about agreement during their interviews. For example, in a run of Party Quirks, B4 made an offer to B2 about the video game Mario Brothers, and B2 accepted it simply by saying, "Yes." B2 later explained,

"I know he knows more about Super Mario than I do. So I'm just thinking, okay, I gotta yes-and him one hundred per cent because I don't have a clue, because I've never played Super Mario." 
This particular acceptance bears similarities to Johnstone's concept of "wimping" [12, p. 114-115], also defined by the Improv Encyclopedia as "accepting offers but refusing to do anything with it" [11].

Although acceptance is considered a basic rule of improv, in practice we saw that improvisers may bend this rule on occasion. For example, in a run of Game, D1 made an offer to D2 that he had a tray of organic muffins, but D2 did not immediately react to this offer. Waiting to come on stage, D3 later recalled,

"The Colombian organic Fair Trade muffins was a ridiculous idea to me, so I was like, okay. He [D1] seemed like-he was pushing the muffins, and making a hard sell on the muffins, I guess, and didn't know if D2 was really buying it or not."

D3 responded by entering the scene as a Colombian woman bearing muffins, thus accepting D1's offer despite D2's perceived hesitation. Thus, acceptance is a complex concept. Improvisers may choose not to accept offers when they believe it is warranted, and other improvisers may accept offers not explicitly intended for them.

\section{DISCUSSION}

This paper reports several aspects of cognition in improvisation: basic cognition, shared mental models, narrative development, and referent use. What is not yet clear is how these different findings can be synthesized into a more singular, comprehensive viewpoint. We intend to explore the interrelationships between these different, bottom-up views, and come to a better understanding of how improvisers make their moment-tomoment decisions as a scene unfolds. For instance, an improviser can have an internal model of what is happening in the narrative of the scene, a hypothesis about what another improviser is attempting to do to move the story forward, and has a suite of improv techniques that are relevant at the moment. Understanding how all of knowledge is used in the same decision process from a top down view is critical to our success in building digital improvisational characters.

The work here represents a full year of effort from over a dozen professors, graduate students, and undergraduates. The cost in designing the experiments, running experimental sessions, and building a working theory have been quite high given the richness of the data we are collecting. What we present here is only the highlights of the working theory we have so far constructed. We have focused our analysis on aspects of cognition and improvisation that will potentially best translate to the creation of computational models of improvisation.

Our selection of experiments was designed to target specific areas of data in both novice, intermediate, and expert runs. We have succeeded in our coverage of the intended space and are now anticipating future game designs that dig deeper into specific data areas. For example, we have found a rich amount of data in our runs of Game, the improv game that focuses on constraints and narrative construction. We have postulated executing more runs of Game where the same functional and content constraints are given to performers over and over again, exhausting the possibilities of that scene. By running that scene multiple times, we can see the different narrative possibilities that exist for the performers, which would not be visible in a single run. Another consideration is doing more "guessing games", like Party Quirks, so we can explore shared mental models in improvisation more deeply

Our current work involve focusing on the different data spaces that we have identified (e.g. narrative development), creating a rigorous coding scheme for each space, and then coding the video data. This will allow us to begin to delve into questions about novice / expert differences in improvisation, the frequency of usage of different improv tactics, etc. This work will allow us to explore cognition and improvisation in a broad and deep scale not seen before in any domain. We hope that by studying the domain of improvisational theatre, this work will show similarities and differences to higher-level findings in other fields (e.g. jazz) and provide data to examine current basic models of how people improvise, such as Johnson-Laird's hybrid model [12]. It is our hope that this work will lead to a more fundamental understanding of how humans conduct improvisational problem solving, what knowledge is brought to bear during that process, etc., so that we are one step closer to understanding human creativity and cognition.

\section{ACKNOWLEDGEMENTS}

Thanks goes to the National Science Foundation for funding this work and to Michael Downing, Kathryn Farley, Dave Joyner, Kate Rosier, and the others who helped conduct the experiments, provide moral support, and contribute to the early design conversations of our methodology.

\section{REFERENCES}

1. Alterhaug, Bjorn. (2004). Improvisation on a Triple Theme: Creativity, Jazz Improvisation, and Communication. Studia Musicologica Norvegica, vol, 30, pp. 97-118.

2. Anderson, J. R. (2005). Cognitive psychology and its implications (Sixth Edition). New York: Worth.

3. Boden, M.A. (1990). The Creative Mind: Myths and Mechanisms. New York: Routledge.

4. Cannon-Bowers, J.A., Salas, E., and Converse, S. (2001). "Shared Mental Models in Expert Team Decision-Making." In Sternberg, R.J. and Grigorenko, E. (Eds.) Environmental Effects of Cognitive Abilities, 221-245.

5. Chatman, S. (1980) Story and Discourse: Narrative Structure in Fiction and Film. Ithaca, New York: Cornell University Press.

6. Clark, H.H., Schaefer, E.F. "Contributing to Discourse." In Cognitive Science: A Multidisciplinary Journal 13.2 (1989) 259-294. 
7. Halpern, C., Close, D., and Johnson, K. (1994). Truth in Comedy: The Manual of Improvisation. Colorado Springs, Colorado: Meriwether Publishing Ltd.

8. Harrell, D. F. (2007). "GRIOT's Tales of Haints and Seraphs: A Computational Narrative Generation System," in Second Person: Role-Playing and Story in Games and Playable Media, N. Wardrip-Fruin and P. Harrigan, Eds. Cambridge:MIT Press.

9. Hayes-Roth, B. and van Gent, R. (1996). StoryMaking with Improvisational Puppets and Actors. Technical Report KSL-96-09, Knowledge Systems Laboratory, Stanford University, Palo Alto, CA.

10. Improv Encyclopedia. (2009). "Offer." Improv Encyclopedia. Accessed April 22, 2009, from $<$ http://improvencyclopedia.org/glossary/Offer.html>

11. Improv Encyclopedia. (2009). "Wimping." Improv Encyclopedia. Accessed April 22, 2009, from $<$ http://improvencyclopedia.org/glossary/Wimping.ht $\mathrm{ml}>$.

12. Johnson-Laird, P.N. (2002). How Jazz Musicians Improvise. Music Perception, 19(3), 415-442.

13. Johnstone, K. (1992). Impro: Improvisation and the Theatre. New York, New York: Routledge / Theatre Arts Books.

14. Johnstone, K. (1999). Impro for Storytellers. New York, New York: Routledge/Theatre Arts Books.

15. Mendonça, D. and Wallace, W. (2004). Cognition in Jazz Improvisation: An Exploratory Study. $26^{\text {th }}$ Annual Meeting of the Cognitive Science Society, Chicago, IL.

16. Mendonça, D. and Wallace, W. (2007). A Cognitive Model of Improvisation in Emergency Management. IEEE Transactions on Systems, Man and Cybernetics - Part A: Systems and Humans, 37(4), pp. 547-561.

17. Mathieu, J.E., Heffner, T.S., Goodwin, G.F., Salas, E., and Cannon-Bowers, J.A. (2002). "The Influence of Shared Mental Models on Team Process and Performance." In Journal of Applied Psychology 85.2, pp. 273-283.

18. Mitroff, I.I., Emshoff, J.R. (1979). "On Strategic Assumption-Making: A Dialectical Approach to Policy and Planning." In Academy of Management Review, 4.1, pp. 1-12.

19. Mohammed, S. (2001). "Toward an Understanding of Cognitive Consensus in a Group Decision-Making Context." In Journal of Applied Behavioral Science 37.4, pp. 408-425.

20. Mohammed, S. and Ringseis, E. (2001). "Cognitive Diversity and Consensus in Group decision Making: The Role of Inputs, Processes, and Outcomes." In Organizational behavior and Human Decision Processes 85.2, pp. 310-335.

21. Newell, A. (1990). Unified Theories of Cognition. Harvard University Press, Cambridge, MA.Pressing, J. (1998). Psychological Constraints on Improvisation. In B. Nettl and M.Russell (Eds.) In the Course of Performance, Studies in the World of Musical Improvisation, 47-67.

22. Pressing, J. (2001). "Improvisation: Methods and Models" in Generative Processes in Music, ed. Sloboda, J., Oxford University Press.

23. Prince, G. (2003) Dictionary of Narratology. Nebraska, University of Nebraska Press.

24. Reinholdsson, R. (1998). Making Music Together: An Interactionist Perspective on Small-Group Performance in Jazz. Acta Universitatis Upsaliensis: Studia Musicologica Upsaliensia, Nova Series 14.

25. Riedl, M.O. and Young, R.M. (2006). Story Planning as Exploratory Creativity: Techniques for Expanding the Narrative Search Space. New Generation Computing, 24(3).

26. Rimmon-Kenan, S. (2002) Narrative Fiction: Contemporary Poetics 2nd Edition. New York, New York: Routledge Taylor \& Francis Group.

27. Ryan, M. (1991) Possible Worlds, Artificial Intelligence, and Narrative Theory. Bloomington, Indiana University Press.

28. Sarath, E. (1996). A New Look at Improvisation. Journal of Music Theory, 40(1), pp. 1-38.

29. Sawyer, K. (2007). Group Genius: The Creative Power of Collaboration. New York: Basic Books.

30. Schank, R. (1988). Creativity as a Mechanical Process. In R.J. Sternberg (Ed.) The Nature of Creativity: Contemporary Psychological Perspectives. $\quad$ Cambridge, U.K.: Cambridge University Press.

31. Schön, R. (1983). The Reflective Practitioner: How Professionals Think in Action. Basic Books, Inc.

32. Spolin, V. (1963). Improvisation for the theater; a handbook of teaching and directing techniques. Evanston, IL: Northwestern University Press.

33. Swartjes, I and Vromen, J. (2007). Emergent Story Generation: Lessons from Improvisational Theatre. In: Proceedings of the AAAI Fall Symposium on Intelligent Narrative Technologies, 9-11 November 2007, Arlington VA

34. Traum, D.R. (1999). "Computational Models of Grounding in Collaborative Systems." In Proceedings of the AAAI Fall Symposium on psychological models of communication pp. 124-131.

35. Wallis, M. \& Shepherd, S. (2002) Studying Plays: Second Edition. Madison Avenue, New York: Oxford University Press Inc.

36. Weick, K.E. (1998). Introductory Essay: Improvisation as a Mindset for Organizational Analysis. Organization Science, 9(5), pp. 543-555.

37. Yokochi, S. and Okada, T. (2005). Creative Cognitive Process of Art Making: A Field Study of a Traditional Chinese Ink Painter. Creativity Research Journal, 17 (2\&3), pp. 241-255. 